\title{
Criopreservação do sêmen de curimba (Prochilodus lineatus) mediante adição de diferentes diluidores, ativadores e crioprotetores ${ }^{1}$
}

\section{Luis David Solis Murgas ${ }^{2}$, Aléssio Batista Miliorini ${ }^{2}$, Rilke Tadeu Fonseca de Freitas ${ }^{3}$, Gilmara Junqueira Machado Pereira²}

\footnotetext{
1 Apoio: Companhia Energética de Minas Gerais - CEMIG.

2 Depto. Medicina Veterinária - Universidade Federal de Lavras, Caixa Postal 37, CEP: 37200-000.

3 Depto. Zootecnia - Universidade Federal de Lavras, Caixa Postal 37, CEP: 37200-000.
}

RESUMO - Amostras de sêmen de cinco espécimes de Prochilodus lineatus (Valencienes, 1847) foram utilizadas para avaliação dos efeitos tóxicos e crioprotetores de seis soluções à base de BTS (Beltsville Thawing Solution ${ }^{\circledR}$ ) $4,5 \%$ enriquecidas com metanol e DMSO nas concentrações de 10\% (A = BTS 4,5\% + Metanol 10\%; B = BTS 4,5\% + DMSO 10\%; C = BTS 4,5\% $+\mathrm{KCl} 0,072 \%+$ metanol 10\%; D $=\mathrm{BTS} 4,5 \%+\mathrm{KCl} 0,072 \%+\mathrm{DMSO} 10 \% ; \mathrm{E}=\mathrm{BTS} 4,5 \%+\mathrm{KI} 0,036 \%+\operatorname{metanol} 10 \%$; e $\mathrm{F}=\mathrm{BTS} 4,5 \%+\mathrm{KI} 0,036 \%+$ DMSO 10\%). Foram avaliadas ainda três soluções ativadoras (água destilada, $\mathrm{NaHCO}_{3} 60$ mM e $\mathrm{NaHCO}_{3} 119 \mathrm{mM}$ ), antes e após o congelamento. Estudaram-se a taxa e a duração da motilidade espermática. Não houve diferença significativa entre as soluções crioprotetoras utilizadas. O sêmen ativado por $\mathrm{NaHCO}_{3} 60$ e 119 mM apresentou as maiores taxas de motilidade espermática. A ativação por $\mathrm{NaHCO}_{3} 119 \mathrm{mM}$ possibilitou as maiores durações de motilidade no sêmen de $P$. lineatus.

Palavras-chave: bicarbonato, congelamento, curimbatá, DMSO, espermatozóides, metanol

\section{Cryopreservation of curimba (Prochilodus lineatus) semen after addition of different diluters, activators and cryoprotectants}

\begin{abstract}
Semen samples of five specimens of Prochilodus lineatus (Valencienes, 1847) were used to test the toxic and cryoprotectants effects of six solutions based on BTS (Beltsville Thawing Solution ${ }^{\circledR}$ ) $4.5 \%$ : A - BTS $4.5 \%+$ Methanol 10\%; B - BTS 4.5\% + DMSO 10\%; C - BTS 4.5\% + KCl $0.072 \%+$ Methanol 10\%; D - BTS 4.5\% + KCl $0.072 \%$ + DMSO 10\%; E - BTS $4.5 \%$ + KI $0.036 \%$ + Methanol $10 \%$ and F - BTS $4.5 \%$ + KI $0.036 \%$ + DMSO $10 \%)$. The effect of other three activator solutions (Distillated Water, $\mathrm{NaHCO} 360 \mathrm{mM}$ and $\mathrm{NaHCO}_{3} 119 \mathrm{mM}$ ) on the rate and duration of sperm motility were also evaluated before and after freezing. No significant differences were observed among the cryoprotectant solutions. Sperm motility rates for $P$. lineatus were higher for semen activated by $\mathrm{NaHCO}_{3} 60 \mathrm{mM}$ and $\mathrm{NaHCO}_{3} 119 \mathrm{mM}$, which propitiated the highest sperm motility duration.
\end{abstract}

Key Words: bicarbonate, curimbatá, DMSO, freezing, methanol, spermatozoids

\section{Introdução}

A capacidade técnica para preservação de gametas e embriões de peixes e de invertebrados aquáticos tem se expandido rapidamente nos últimos anos, impulsionada primariamente pela indústria aqüícola (Carolsfeld et al., 2003). A criopreservação é uma técnica importante no controle reprodutivo de muitas espécies e sua aplicação em peixes está em amplo desenvolvimento. Envolve procedimentos que permitem o armazenamento de espermatozóides em nitrogênio líquido, mantendo-se a viabilidade dos gametas por tempo indefinido. Mins et al. (2000) verificaram que a porcentagem de espermatozóides móveis contidos no sêmen criopreservado de esturjão não diminuiu ao longo de cinco anos de armazenamento. A criopreservação constitui técnica importante na rotina da reprodução induzida e na melhoria genética de espécies de peixe por aumentar as chances de se desenvolverem pesquisas, sobretudo no campo genético, sendo indicada para minimizar a assincronia na maturação dos gametas, no uso seletivo de reprodutores ou mesmo no transporte de gametas (Godinho, 2000). A criopreservação de células ganhou impulso quando pesquisas relataram que o glicerol possui capacidade de protegê-las contra efeitos deletérios do congelamento. Desde então, várias substâncias vêm sendo testadas com esse objetivo (Godinho, 2000). Segundo Harvey (1993), o metanol é a substância que mais facilmente permeia a membrana celular, sendo, entretanto, considerado a mais 
tóxica, exceto para o sêmen de tilápia (Oreochromus niloticus). Bedore (1999) relatou elevada toxicidade do dimetilsulfóxido (DMSO) para o sêmen de peixes, porém, alguns autores, como Miliorini et al. (2002), não encontraram diferenças entre as concentrações de 5 e $10 \%$ de DMSO em até 72 horas de resfriamento $\left(4^{\circ} \mathrm{C}\right)$ do sêmen de pacu (Piaractus mesopotamicus) - inclusive, as maiores taxas de motilidade até 96 horas ocorreram na maior concentração. Outros autores observaram que o DMSO é um dos melhores crioprotetores para espécies de peixes de água doce (Bedore, 1999) e de água salgada (Ritar, 1999) e que sua toxicidade pode ser suprida pela adição de carboidratos (Leung \& Jamielson, 1991).

Do mesmo modo que a utilização de crioprotetores é importante no sucesso de preservação criogênica de sêmen de peixes, a incorporação de soluções diluidoras é fundamental para proporcionar meio osmótico e nutricionalmente ideal aos espermatozóides durante a aplicação da biotecnologia. O BTS (Beltsville Thawing Solution ${ }^{\circledR}$ ) é um diluidor desenvolvido e preconizado para conservação do sêmen suíno. Entretanto, vários autores (Franciscatto et al., 2002; Miliorini et al., 2002; Murgas et al., 2002; Maria et al., 2004; Miliorini et al., 2004) têm utilizado com sucesso esse diluidor em pesquisas com peixes de piracema. O BTS possui em sua fórmula componentes que, além de nutrirem células espermáticas e proporcionarem um microambiente osmoticamente favorável, protegem a face intracelular da membrana citoplasmática durante o congelamento.

Entre as soluções ativadoras da motilidade espermática, merecem destaque a água destilada e as soluções salinas de bicarbonato de sódio, $\mathrm{NaCl}$ e $\mathrm{KCl}$ em diferentes concentrações, dependendo da espécie (Ravinder et al., 1997). Lahnesteiner et al. (1995) verificaram no sêmen de curimatã (Prochilodus argentus) que, em relação à água destilada, soluções de $\mathrm{NaCl}$, utilizadas como ativadoras da motilidade espermática, aumentaram o vigor espermático, apesar de reduzirem sua duração. Para o sêmen de pacu $(P$. mesopotamicus), Fogli da Silveira et al. (1995), citados por Cruz (2001), estimaram os parâmetros de motilidade utilizando solução de $\mathrm{NaHCO}_{3}$, ao passo que Miliorini et al. (2004) utilizaram $\mathrm{NaCl}(30$ e $60 \mathrm{mM})$ e $\mathrm{NaHCO}_{3}(30$ e $60 \mathrm{mM})$ como soluções ativadoras do sêmen de $P$. lineatus resfriado. Nestes trabalhos, as soluções com as concentrações mais elevadas dos sais proporcionaram taxas de motilidade espermática também superiores.

A espécie $P$. lineatus é muito apreciada na culinária nordestina e sua comercialização tem sido promissora, sobretudo no Nordeste do Brasil. Considerando o grande impacto que a biotecnologia de criopreservação de gametas exerce sobre inúmeros aspectos (reprodutivo, ecológico, social e econômico) e a escassez de trabalhos sobre a reprodução de espécies de piracema, realizou-se este estudo com o objetivo de estudar os efeitos da utilização de diferentes diluidores, crioprotetores e soluções ativadoras sobre as características de motilidade espermática após o processo de criopreservação do sêmen de $P$. lineatus.

\section{Material e Métodos}

Foram utilizados cinco machos de $P$. lineatus com

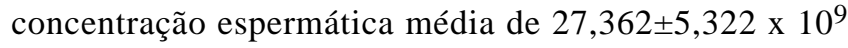
espermatozóides. $\mathrm{mL}^{-1}$ e 1.160,0 $4487,8 \mathrm{~g}$ de peso vivo, capturados do leito do Rio Grande, no município de Itutinga, MG, às margens da usina hidroelétrica de Camargos. Os animais foram mantidos como parte do plantel da Unidade Ambiental da Companhia Energética de Minas Gerais (CEMIG), localizada no mesmo município, sendo selecionados segundo as características: coloração eritematosa e aspecto edemaciado da papila urogenital e eliminação de sêmen em resposta à massagem manual sobre a parede celomática. Os exemplares foram transportados até o laboratório de reprodução, onde foram pesados, dimensionados e acondicionados em tanques de tratamento para isolamento de fêmeas e de exemplares de outras espécies.

Os animais utilizados receberam duas injeções intramusculares de extrato bruto de hipófise de carpa (EBHC), nas doses de 0,4 e 4,0 mg/kg de peso corporal, em intervalo de aproximadamente 12 horas. A última dose, por seu turno, antecedeu 9 a 10 horas o período da coleta.

Para coleta individual do ejaculado, utilizou-se um puçá para a emersão dos animais, que foram contidos por uma toalha de algodão umedecida. Os olhos foram vendados e a papila urogenital foi limpa e seca com toalha de papel. Efetuaram-se compressões manuais da parede celomática, no sentido crânio-caudal. O sêmen foi obtido livre de fezes, urina, sangue ou água. Quando ativado precocemente, o sêmen foi descartado. Os ejaculados foram coletados em tubos de ensaio opacos, sendo acondicionados e imersos em gelo, ao abrigo da luz, para posterior análise laboratorial.

A análise da qualidade do sêmen fresco (e demais análises subseqüentes) de cada macho utilizado foi realizada em uma pequena gota depositada sobre lâmina de microscopia e observada ao microscópio de luz, previamente focalizado em aumento de 400X. A observação dos espermatozóides imóveis permitiu avaliar a pureza do sêmen. A seguir, o sêmen foi ativado mediante adição de água do próprio tanque para avaliação de sua qualidade e viabilidade para ser criopreservado. Todos os ejaculados 
utilizados se mostraram completamente $(100 \%$ de espermatozóides móveis) aptos ao congelamento. Foram aferidas a taxa (\%) e a duração (segundos) da motilidade espermática. A duração da motilidade foi considerada desde a ativação até que apenas 10\% das células espermáticas apresentassem motilidade.

Dois experimentos foram conduzidos para avaliação dos componentes do protocolo de criopreservação. No experimento 1 , procedeu-se à avaliação da toxicidade dos crioprotetores e diluidores mediante ativação espermática por diferentes ativadores. Foram adicionadas lentamente às amostras seis soluções (na proporção de uma parte de sêmen para quatro partes de solução), cada uma previamente preparada (estabilizada) e composta do seguinte modo:

$\S$ Solução A: BTS (TAB.1) 4,5\% + Metanol 10\% (concentração final);

$\S$ Solução B: BTS 4,5\% + DMSO 10\% (concentração final);

§ Solução C: BTS 4,5\% + KCl 0,072\% (concentração final) + Metanol 10\%;

§ Solução D: BTS 4,5\% + KCl 0,072\% + DMSO 10\%;

$\S$ Solução E: BTS 4,5\% + KI 0,036\% (concentração final) + Metanol 10\%; e

§ SoluçãoF: BTS 4,5\% + KI 0,036\% + DMSO 10\%

A razão da adição de solutos ( $\mathrm{KCl}$ e KI, nas concentrações finais indicadas) às soluções $\mathrm{C}$ a $\mathrm{F}$ é justificada em trabalhos anteriores, realizados por Franciscatto et al. (2002), Miliorini et al. (2002) e Murgas et al. (2002). De acordo com esses autores, o diluidor BTS deve ser acrescido de íons e seu uso não resultou em ativação dos espermatozóides quando do resfriamento do sêmen de pacu (P. mesopotamicus) e piapara (Leporinus obtusidens).

Em uma lâmina de microscopia, previamente focalizada, colocou-se uma alíquota de aproximadamente $1 \mu \mathrm{L}$ da mistura homogeneizada sêmen fresco + soluções (A a F). A seguir, procedeu-se à ativação da motilidade testando-se três soluções ativadoras (água destilada; $\mathrm{NaHCO}_{3} 60 \mathrm{mM}$; e $\mathrm{NaHCO}_{3} 119 \mathrm{mM}$ ) por meio da adição de $6 \mu \mathrm{L}$ de solução ativadora. Após a homogeneização da mistura, foram determinadas a taxa e a duração da motilidade espermática. Todo o procedimento foi conduzido em temperatura ambiente $\left(23^{\circ} \mathrm{C}\right)$ simulando condições de campo.

O experimento 2 consistiu da avaliação do efeito das soluções A a F sobre a motilidade espermática após congelamento. Imediatamente após a diluição, as amostras foram envasadas em palhetas de $0,5 \mathrm{~mL}$ de capacidade, gerando um total de quatro palhetas/amostra de sêmen. As palhetas foram transferidas para um botijão de vapor de nitrogênio
Tabela 1 - Composicão química do diluidor Beltsville Thawing Solution ${ }^{\circledR}$ - BTS $(100 \mathrm{~g})$

Table 1 - Chemical composition of Beltsville Thawing Solution ${ }^{\circledR}$ - BTS (100 g)

\begin{tabular}{lr}
\hline $\begin{array}{l}\text { Constituinte } \\
\text { Constituent }\end{array}$ & $\begin{array}{c}\text { Quantidade (g) } \\
\text { Amount }\end{array}$ \\
\hline Glicose (Glucose) & 79,90 \\
Citrato de sódio (Sodium citrate) & 12,71 \\
EDT A & 2,65 \\
Bicarbonato de sódio (Sodium bicarbonate) & 2,65 \\
Cloreto de potássio (Potassium chlorate) & 1,59 \\
Sulfato de gentamicina (Gentamicyn sulphate) & 0,50
\end{tabular}

${ }^{\circledR}$ MINITÜB ABFÜLL-UND LABORTECHNIK Gmbh \& Co.KG

líquido (Taylor-Wharton, modelo CP 300, tipo dryshipper) e mantidas em posição vertical. Vinte e quatro horas depois, foram submersas em nitrogênio líquido em botijão de armazenamento (Cryometal, modelo DS-18) a uma temperatura estável de $-96^{\circ} \mathrm{C}$. O descongelamento das amostras de sêmen foi feito após período igual ou superior a 1 hora de congelamento. O descongelamento envolveu a retirada das palhetas do botijão e sua imersão em água a $60^{\circ} \mathrm{C}$ por oito segundos. A seguir, procederam-se às análises da taxa $\mathrm{e}$ duração da motilidade espermática segundo o procedimento descrito para o experimento 1 .

Para a análise da concentração espermática, diluiu-se uma alíquota de $1 \mu \mathrm{L}$ de sêmen em $1 \mathrm{~mL}$ de solução de formol citrato. O número de espermatozóides. $\mathrm{mL}^{-1}$ foi estimado por meio de exame direto em câmara hematimétrica.

Os experimentos foram conduzidos em delineamento em blocos casualizados (DBC), com os tratamentos em arranjo fatorial $3 \times 2 \times 3$ (diluidores $\mathrm{x}$ crioprotetores $\mathrm{x}$ soluções ativadoras) com seis repetições (blocos), em que o peixe constituiu o bloco.

Os dados obtidos foram submetidos à análise estatística por meio do Sistema de Análise de Variância - SISVAR (Ferreira, 1999), de acordo com o seguinte modelo:

$$
\begin{gathered}
Y_{i j k l}=\mu+B_{1}+D_{i}+C_{j}+A_{k}+D_{i j}+D_{i k}+C_{j k}+ \\
D C A_{i j k}+E_{i j k l}
\end{gathered}
$$

em que $\mathrm{Y}_{\mathrm{ijk} 1}=$ observação 1 obtida para o diluidor $\mathrm{i}$, crioprotetor $\mathrm{j}$ e solução ativadora $\mathrm{k} ; \mu=$ constante associada a cada observação; $\mathrm{B}_{1}=$ efeito do bloco $1,1=1,2, \ldots$, 6; $\mathrm{D}_{\mathrm{i}}=$ efeito do diluidor $\mathrm{i}, \mathrm{i}=1,2$ e $3 ; \mathrm{C}_{\mathrm{j}}=$ efeito do crioprotetor $\mathrm{j}, \mathrm{j}=1$ e $2 ; \mathrm{A}_{\mathrm{k}}=$ efeito da solução ativadora $\mathrm{k}$, $\mathrm{k}=1,2$ e $3 ; \mathrm{DC}_{\mathrm{ij}}=$ efeito da interação diluidor $\mathrm{i} \times$ crioprotetor $\mathrm{j} ; \mathrm{DA}_{\mathrm{ik}}=$ efeito da interação diluidor $\mathrm{i} \times$ solução ativadora $\mathrm{k} ; \mathrm{CA}_{\mathrm{jk}}=$ efeito da interação crioprotetor $\mathrm{j} \times$ solução ativadora $\mathrm{k} ; \mathrm{DCA}_{\mathrm{ijk}}=$ efeito da interação diluidor $\mathrm{i} \times$ crioprotetor $\mathrm{j} \times$ solução ativadora $\mathrm{k} ; \mathrm{E}_{\mathrm{ijkl}}=$ erro associado a cada observação, que, por pressuposição, é $\operatorname{NID}\left(0, \delta^{2}\right)$ 
As médias foram comparadas pelo teste de StudentNewman-Keuls (nível mínimo de significância=0,01).

\section{Resultados e Discussão}

A análise de toxicidade (experimento 1) comprovou não haver diferenças significativas entre as soluções contendo os diferentes crioprotetores $(\mathrm{P}>0,05)$. Como demonstrado nas Tabelas 2 e 3 , todas as soluções utilizadas possibilitaram taxas e durações semelhantes depois de acrescentadas ao sêmen.
Silva (2000) utilizou DMSO 10\% e gema de ovo como crioprotetores para o sêmen da mesma espécie, contudo, apesar de a taxa de motilidade pré-congelamento ter sido superior a $80 \%$ após 2 horas de congelamento, a uma temperatura de descongelamento de $26^{\circ} \mathrm{C}$ por 10 segundos, tanto a taxa como a duração da motilidade espermática se mostraram nulas, indicando mortalidade total dos espermatozóides. Os resultados encontrados neste estudo foram semelhantes aos verificados por Miliorini et al. (2004), que verificaram que o DMSO apresentou toxicidade para o sêmen de $P$. lineatus similar ao metanol e ao etilenoglicol

Tabela 2 - Taxas de motilidade (\%) do sêmen de $P$. lineatus diluído em diferentes soluções antes do congelamento Table 2 - Spermatic motility rates (\%) of $P$. lineatus semen diluted in different solutions before freezing

\begin{tabular}{|c|c|c|c|c|}
\hline \multirow{3}{*}{$\begin{array}{l}\text { Solução } \\
\text { Solution }\end{array}$} & \multirow{2}{*}{\multicolumn{3}{|c|}{$\begin{array}{l}\text { Ativador } \\
\text { Activator }\end{array}$}} & \multirow{3}{*}{$\begin{array}{l}\text { Média } \\
\text { Mean }\end{array}$} \\
\hline & & & & \\
\hline & $\begin{array}{l}\text { Água destilada } \\
\text { Distillated water }\end{array}$ & $\begin{array}{l}\text { Bicarbonato de sódio } \\
\qquad 60 \mathrm{mM} \\
\text { Sodium bicarbonate }\end{array}$ & $\begin{array}{l}\text { Bicarbonato de sódio } \\
119 \mathrm{mM} \\
\text { Sodium bicarbonate }\end{array}$ & \\
\hline $\begin{array}{l}\text { BTS } 4,5 \%+\text { Metanol } 10 \% \\
\text { BTS } 4.5 \%+\text { Methanol } 10 \%\end{array}$ & $85 \pm 11,18$ & $94 \pm 4,18$ & $93 \pm 2,73$ & $90 \pm 7,76^{a}$ \\
\hline $\begin{array}{l}\text { BTS } 4,5 \%+\text { DMSO } 10 \% \\
\text { BTS } 4.5 \%+\text { DMSO } 10 \%\end{array}$ & $73 \pm 19,23$ & $86 \pm 10,83$ & $81 \pm 24,59$ & $80 \pm 18,51^{a}$ \\
\hline $\begin{array}{l}\text { BTS } 4,5 \%+\mathrm{KCl} 0,072 \%+\text { Metanol } 10 \% \\
\text { BTS } 4.5 \%+\mathrm{KCl} 0.072 \%+\text { Methanol } 10 \%\end{array}$ & $70 \pm 23,45$ & $93 \pm 5,70$ & $92 \pm 10,36$ & $85 \pm 17,82^{a}$ \\
\hline $\begin{array}{l}\mathrm{BTS} 4,5 \%+\mathrm{KCl} 0,072 \%+\mathrm{DMSO} 10 \% \\
\text { BTS } 4.5 \%+\mathrm{KCl} 0.072 \%+\text { DMSO } 10 \%\end{array}$ & $64 \pm 22,19$ & $88 \pm 10,36$ & $92 \pm 8,36$ & $81 \pm 18,84^{a}$ \\
\hline $\begin{array}{l}\text { BTS } 4,5 \%+\text { KI } 0,036 \%+\text { Metanol } 10 \% \\
\text { BTS } 4.5 \%+K C l 0.036 \%+\text { Methanol } 10 \%\end{array}$ & $70 \pm 28,50$ & $81 \pm 13,41$ & $85 \pm 11,18$ & $78 \pm 19,03^{a}$ \\
\hline $\begin{array}{l}\text { BTS } 4,5 \%+\text { KI } 0,036 \%+\text { DMSO } 10 \% \\
\text { BTS } 4.5 \%+K C l 0.036 \%+D M S O 10 \%\end{array}$ & $71 \pm 8,94$ & $90 \pm 11,72$ & $92 \pm 13,03$ & $84 \pm 14,37^{a}$ \\
\hline $\begin{array}{l}\text { Média } \\
\text { Average }\end{array}$ & $72 \pm 19,41$ & $88 \pm 10,08$ & $89 \pm 13,07$ & $83 \pm 16,57$ \\
\hline
\end{tabular}

a Médias não diferem $(P>0,05)$ pelo teste Student-Newman-Keuls.

${ }^{a}$ Averages followed by the same letters do not differ $(P>0.05)$ by Student Newman-Keuls test.

Tabela 3 - Duração da motilidade (segundos) do sêmen de $P$. lineatus diluído em diferentes soluções antes do congelamento Table 3 - Duration of spermatic motility (seconds) of $P$. lineatus semen diluted in different solutions before freezing

\begin{tabular}{|c|c|c|c|c|}
\hline \multirow[t]{2}{*}{$\begin{array}{l}\text { Solução } \\
\text { Solution }\end{array}$} & \multicolumn{3}{|c|}{$\begin{array}{l}\text { Ativador } \\
\text { Activator }\end{array}$} & \multirow[t]{2}{*}{$\begin{array}{l}\text { Média } \\
\text { Mean }\end{array}$} \\
\hline & $\begin{array}{l}\text { Água destilada } \\
\text { Distillated water }\end{array}$ & $\begin{array}{l}\text { Bicarbonato de sódio } \\
60 \mathrm{mM} \\
\text { Sodium bicarbonate }\end{array}$ & $\begin{array}{l}\text { Bicarbonato de sódio } \\
119 \mathrm{mM} \\
\text { Sodium bicarbonate }\end{array}$ & \\
\hline $\begin{array}{l}\text { BTS } 4,5 \%+\text { Metanol } 10 \% \\
\text { BTS } 4.5 \%+\text { Methanol } 10 \%\end{array}$ & $37 \pm 8,30$ & $159 \pm 102,20$ & $131 \pm 63,67$ & $109 \pm 91,08^{a}$ \\
\hline $\begin{array}{l}\text { BTS } 4,5 \%+\text { DMSO } 10 \% \\
\text { BTS } 4.5 \%+\text { DMSO } 10 \%\end{array}$ & $37 \pm 14,38$ & $161 \pm 153,78$ & $109 \pm 54,84$ & $101 \pm 92,16^{a}$ \\
\hline $\begin{array}{l}\text { BTS } 4,5 \%+\mathrm{KCl} 0,072 \%+\text { Metanol } 10 \% \\
\text { BTS } 4.5 \%+\mathrm{KCl} 0.072 \%+\text { Methanol } 10 \%\end{array}$ & $33 \pm 9,73$ & $72 \pm 32,60$ & $141 \pm 126,21$ & $82 \pm 63,79^{a}$ \\
\hline $\begin{array}{l}\mathrm{BTS} 4,5 \%+\mathrm{KCl} 0,072 \%+\text { DMSO } 10 \% \\
\text { BTS } 4.5 \%+\mathrm{KCl} 0.072 \%+\text { DMSO } 10 \%\end{array}$ & $29 \pm 16,72$ & $51 \pm 22,61$ & $124 \pm 57,62$ & $68 \pm 54,02^{a}$ \\
\hline $\begin{array}{l}\text { BTS } 4,5 \%+\text { KI } 0,036 \%+\text { Metanol } 10 \% \\
\text { BTS } 4.5 \%+K C l 0.036 \%+\text { Methanol } 10 \%\end{array}$ & $33 \pm 19,94$ & $72 \pm 10,82$ & $171 \pm 101,55$ & $92 \pm 79,42^{\mathrm{a}}$ \\
\hline $\begin{array}{l}\text { BTS } 4,5 \%+\text { KI } 0,036 \%+\text { DMSO } 10 \% \\
B T S 4.5 \%+K C l 0.036 \%+D M S O 10 \%\end{array}$ & $29 \pm 3,78$ & $82 \pm 34,93$ & $184 \pm 130,38$ & $98 \pm 88,22^{a}$ \\
\hline $\begin{array}{l}\text { Média } \\
\text { Mean }\end{array}$ & $33 \pm 12,52$ & $99 \pm 83,74$ & $142 \pm 108,66$ & $92 \pm 77,24$ \\
\hline
\end{tabular}

a Médias não diferem $(P>0,05)$ pelo teste Student-Newman-Keuls.

a Averages followed by the same letters do not differ $(P>0.05)$ by Student Newman-Keuls test. 
na concentração de 10,0\% após 6 horas de resfriamento a $4^{\circ} \mathrm{C}$. Entretanto, o DMSO propiciou redução na motilidade significativamente superior à obtida com o metanol nas 42 horas seguintes. Segundo Bedore (1999), concentrações ou períodos de estabilização elevados de DMSO podem provocar redução significativa na qualidade do sêmen de peixes.

Cruz (2001) também verificou que as amostras acrescidas de DMSO apresentaram duração média de motilidade superior à daquelas acrescidas de etilenoglicol e propanodiol, sendo a concentração de 15,0\% (104 segundos) a menos tóxica em comparação às concentrações de 5,0 (67 segundos) e $10,0 \%$ ( 84 segundos). $\mathrm{O} \mathrm{NaHCO}_{3}$ tem sido utilizado com sucesso na ativação da motilidade do sêmen de peixes. Miliorini et al. (2002) e Murgas et al. (2002) observaram, respectivamente, que as amostras de sêmen de $P$. mesopotamicus e $L$. obtusidens ativadas com $\mathrm{NaHCO}_{3} 119$ $\mathrm{mM}$ e acrescidas de DMSO 10,0\% apresentaram taxas e durações médias da motilidade superiores às das amostras ativadas por água destilada e acrescidas de DMSO 5,0\%. Aparentemente, amostras de sêmen in natura ou resfriadas ativadas por concentrações inferiores de $\mathrm{NaHCO}_{3}$ não respondem com a mesma qualidade mótil.

Não foi observada diferença significativa entre as soluções crioprotetoras testadas quanto às características estudadas no sêmen descongelado $(\mathrm{P}>0,05)$. Porém, a ativação por $\mathrm{NaHCO}_{3} 60 \mathrm{mM}$ e $119 \mathrm{mM}$ propiciou (Tabela 4) as maiores taxas de motilidade espermática $(\mathrm{P}<0,01)$. A ativação por meio de $\mathrm{NaHCO}_{3} 119 \mathrm{mM}$ possibilitou (Tabela 5) as maiores durações de motilidade espermática em comparação à utilização das soluções de $\mathrm{NaHCO}_{3} 60 \mathrm{mM}$ e água destilada $(\mathrm{P}<0,01)$. A comparação aos estudos de Cruz (2001) e Franciscatto et al. (2002), além

Tabela 4 - Taxas de motilidade (\%) do sêmen de $P$. lineatus ativado com diferentes soluções antes e após o congelamento

Table 4 - $\quad$ Spermatic motility rates (\%) of $P$. lineatus semen activated in different solutions after and before freezing

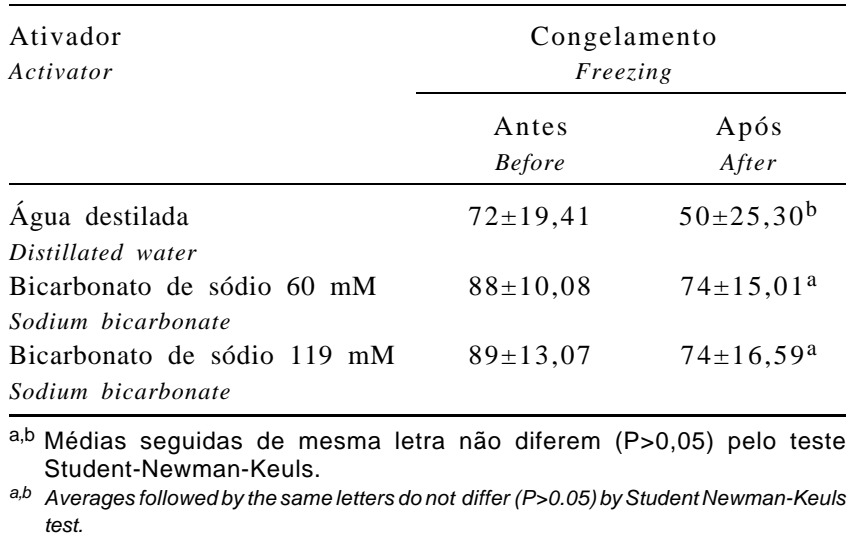

da observação em outras espécies como $P$. mesopotamicus (Miliorini et al., 2002) e L. obtusidens (Murgas et al., 2002), permite sugerir melhor desempenho do ativador $\mathrm{NaHCO}_{3}$ $119 \mathrm{mM}$ à medida que se alteram as temperaturas e/ou se prolonguem os períodos de estocagem do sêmen de $P$. lineatus. Além disso, é possível que o $\mathrm{NaHCO}_{3} 119 \mathrm{mM}$ tenha proporcionado um meio com menor diferença osmótica em relação ao citoplasma, preservando a integridade da membrana citoplasmática dos espermatozóides de $P$. lineatus. Entretanto, é necessário conhecer se a taxa de motilidade proporcionada pelo $\mathrm{NaHCO}_{3} 119 \mathrm{mM}$ após o descongelamento também reflete em elevadas taxas de fertilização e se esse ativador não exerce toxidez sobre os oócitos. Portanto, a aplicabilidade desses resultados deve ser comprovada mediante a realização de ensaios que estimem o potencial fertilizante e de eclodibilidade do sêmen de $P$. lineatus congelado sob os protocolos testados neste trabalho.

O BTS apresenta glicose em sua formulação (Tabela 1), que, por sua vez, atua como crioprotetor externo à membrana citoplasmática dos espermatozóides e serve de substrato para a síntese de ATP. Segundo Cosson et al. (1999), a fosforilação oxidativa mitocondrial é altamente requerida para produção de energia durante o batimento flagelar dos espermatozóides, de modo que a insuficiência de ATP constitui uma das principais causas da redução da motilidade espermática. Lahnsteiner et al. (1993) verificaram que o espermatozóide de $O$. mykiss utiliza triglicerídeos e glicose como fontes primárias de energia. É possível que o sêmen de $P$. lineatus apresente metabolismo semelhante e que a concentração de glicose exógena utilizada neste estudo (em torno de 3,0\%, após a diluição do sêmen) tenha sido suficiente para suprir as necessidades metabólicas das

Tabela 5 - Duração da motilidade (segundos) do sêmen de $P$. lineatus ativado em diferentes soluções antes e após o congelamento

Table 5 - Duration of spermatic motility (seconds) of $P$. lineatus semen activated in different solutions before and after freezing

\begin{tabular}{|c|c|c|}
\hline \multirow[t]{2}{*}{$\begin{array}{l}\text { Ativador } \\
\text { Activator }\end{array}$} & \multicolumn{2}{|c|}{$\begin{array}{l}\text { Congelamento } \\
\text { Freezing }\end{array}$} \\
\hline & $\begin{array}{l}\text { Antes } \\
\text { Before }\end{array}$ & $\begin{array}{l}\text { Após } \\
\text { After }\end{array}$ \\
\hline $\begin{array}{l}\text { Água destilada } \\
\text { Distillated water }\end{array}$ & $33 \pm 12,52$ & $18 \pm 8,25^{b}$ \\
\hline $\begin{array}{l}\text { Bicarbonato de sódio } 60 \mathrm{mM} \\
\text { Sodium bicarbonate }\end{array}$ & $99 \pm 83,74$ & $48 \pm 23,38^{b}$ \\
\hline $\begin{array}{l}\text { Bicarbonato de sódio } 119 \mathrm{mM} \\
\text { Sodium bicarbonate }\end{array}$ & $142 \pm 108,66$ & $73 \pm 41,02^{\mathrm{a}}$ \\
\hline
\end{tabular}

a,b Médias seguidas de mesma letra não diferem $(P>0,05)$ pelo teste Student-Newman-Keuls.

a,b Averages followed by the same letters do not differ $(P>0.05)$ by Student Newman-Keuls test. 
células espermáticas. A utilização do diluidor BTS, portanto, não demanda definitivamente a adição de $\mathrm{KCl}$ ou KI se incrementado dos crioprotetores internos Metanol $10 \%$ e DMSO $10 \%$, tendo em vista o fato de não ativarem precocemente a motilidade espermática antes do congelamento.

\section{Conclusões}

A metodologia utilizada neste trabalho para criopreservação do sêmen de $P$. lineatus foi adequada, pois permitiu a obtenção de taxas de motilidade espermática pós-descongelamento superiores a $70 \%$.

A solução de $\mathrm{NaHCO}_{3} 119 \mathrm{mM}$ pode ser usada como ativador da motilidade do sêmen de $P$. lineatus após descongelamento.

O diluidor BTS pode ser utilizado satisfatoriamente, na concentração final de 4,5\% para a criopreservação do sêmen de $P$. lineatus se, concomitantemente, for enriquecido com os crioprotetores intracelulares metanol ou DMSO em concentrações finais de $10 \%$.

\section{Literatura Citada}

BEDORE, A.G. Características e criopreservação do sêmen de pacu-caranha (Piaractus mesopotamicus) e de piracanjuba (Brycon orbignyanus). Belo Horizonte: Universidade Federal de Minas Gerais, 1999. 53p. Dissertação (Mestrado em Biologia Celular) - Universidade Federal de Minas Gerais, 1999.

CAROLSFELD, J.; HARVEY, B. Conservação de recursos genéticos de peixes: teoria e prática. Curso de Treinamento Brasileiro. Victoria: World Fisheries Trust, 1999. 41p.

CAROLSFELD J.; GODINHO H.P.; ZANIBONI FILHO, E. et al Cryopreservation of sperm in Brazilian migratory fish conservation. Journal of Fish Biology, v.63, n.2, p.472489,2003

COSSON, J.; BILLARD, R.; CIBERT, C. et al. Ionic factors regulating the motility of fish sperm. In: GAGNON, C. (Ed.) The male gamete: from basic science to clinical applications. Vienna: Cache River Press, 1999. p.162-186.

CRUZ, V.L.B. Criopreservação de sêmen de curimbatá (Prochilodus lineatus). Belo Horizonte: Pontifícia Universidade Católica de Minas Gerais, 2001. 59p. Dissertação (Mestrado em Zoologia de Vertebrados) - Pontifícia Universidade Católica de Minas Gerais, 2001.

FERREIRA, D.F. Sistema de análise de variância (Sisvar). versão 4.6. Lavras: Departamento de Ciências Exatas, 1999. (CD-ROM).
FRANCISCATTO, R.T.; MURGAS, L.D.S.; MILIORINI, A.B. et al. Qualidade do sêmen de curimba (Prochilodus lineatus) e taxa de fertilidade após resfriamento a $4^{\circ} \mathrm{C}$. Revista Brasileira de Reprodução Animal, v.26, n.3, p.213-215, 2002.

GODINHO, H.P. Criopreservação de sêmen de peixes. Informe Agropecuário, v.21, n.203, p.6-20, 2000.

HARVEY, B.; CAROLSFELD, J. Preservation of sperm. In: HARVEY, B.; CAROLSFELD, J. (Eds.) Induced breeding in tropical fish culture. Otawa: International Development Research Center, 1993. p.119-130.

LAHNSTEINER, F.; PATZNER, R.A.; WEISMANN, T. Energy resources of spermatozoa of the rainbow trout Oncorhynchus mykiss (Pisces, Teleostei). Reproduction and Nutritional Development, v.33, p.349-360, 1993

LEUNG, L.K.P.; JAMIESON, B.G.M. Live preservation of fish gametes. In: JAMIESON, B.G.M. (Ed.). Fish evolution and systematics: evidence from spermatozoa. Cambridge: Cambridge University Press, 1991. p.245-269.

MARIA, A.N.; MURGAS, L.D.S.; SILVA, M.O.B. et al. Influência da adição de iodeto de potássio e citrato de sódio na qualidade do sêmen de pacu (Piaractus mesopotamicus - Holmberg, 1887). Ciência e Agrotecnologia, v.28, n.1, p.191-195, 2004.

MILIORINI, A.B.; MURGAS, L.D.S.; VIVEIROS, A.T.M. et al. Resfriamento do sêmen de pacu (Piaractus mesopotamicus) a $4^{\circ} \mathrm{C}$, utilizando diferentes concentrações de dimetilsulfóxido. Revista Brasileira de Reprodução Animal, v.26, n.3, p.209$211,2002$.

MILIORINI, A.B.; MURGAS, L.D.S.; VIVEIROS, A.T.M. et al. The effects of cryoprotectants and activators on sperm motility of curimba (Prochilodus lineatus). In: INTERNATIONAL CONGRESS ON ANIMAL REPRODUCTION, 15., 2004, Porto Seguro. Anais... Porto Seguro: 2004. p.523.

MINS, S.D.; TSVETKOVA, L.I.; BROWN, G.G. et al. Cryopreservation of sperm of sturgeon and paddlefish. In: TIERSCH, T.R.; MAZIK, P.M. (Eds). Cryopreservation in aquatic species. Baton Rouge: World Aquaculture Society, 2000. p.123-129.

MURGAS, L.D.S.; MILIORINI, A.B.; SILVA, M.O.B. et al Viabilidade seminal de piapara (Leporinus obtusidens), empregando-se diferentes diluentes, no resfriamento do sêmen a $4^{\circ} \mathrm{C}$. Revista Brasileira de Reprodução Animal, v.26, n.3, p.211-213, 2002

RAVINDER, K.; NASARUNDDIN, K.; MAJUMDAR, K.C. et al. Computerized analysis of motility patterns and parameters of spermatozoa of carp following short-term storage of semen. Journal of Fish Biology, v.50, n.6, p.1309-1328, 1997.

RITAR, A.J. Artificial insemination with cryopreserved semen from striped trumpeter (Latris lineata). Aquaculture Research, v. 180, p.177-187, 1999.

SILVA, E.B. Avaliação comparativa da utilização do sêmen criopreservado e fresco na fertilização dos óvulos de curimatã Prochilodus lineatus (Valenciennes, 1847). Florianópolis: Universidade Federal de Santa Catarina, 2000. 49p. Dissertação (Mestrado em Aqüicultura) - Universidade Federal de Santa Catarina, 2000.

Recebido: 22/08/06 Aprovado: 06/12/06 